\title{
Masers in GLIMPSE Extended Green Objects (EGOs)
}

\section{Claudia J. Cyganowski ${ }^{1}$, Crystal L. Brogan ${ }^{2}$, Todd R. Hunter ${ }^{2}$, Ed Churchwell ${ }^{3}$, Jin Koda ${ }^{4}$, Erik Rosolowsky ${ }^{5}$, Sarah Towers ${ }^{4}$, Barb Whitney ${ }^{3}$, and Qizhou Zhang ${ }^{6}$}

\author{
${ }^{1}$ Harvard-Smithsonian Center for Astrophysics, \\ Cambridge, MA 02138 USA \\ NSF Astronomy and Astrophysics Postdoctoral Fellow \\ email: ccyganowski@cfa.harvard.edu \\ ${ }^{2}$ National Radio Astronomy Observatory, Charlottesville, VA 22902 USA \\ ${ }^{3}$ Department of Astronomy, University of Wisconsin-Madison, Madison, WI, 53706, USA \\ ${ }^{4}$ Department of Physics and Astronomy, Stony Brook University, Stony Brook, NY 11794, USA \\ ${ }^{5}$ Department of Physics and Astronomy, University of British Columbia, Okanagan, Kelowna \\ BC, Canada \\ ${ }^{6}$ Harvard-Smithsonian Center for Astrophysics, Cambridge, MA 02138 USA
}

\begin{abstract}
Large-scale Spitzer surveys of the Galactic plane have yielded a new diagnostic for massive young stellar objects (MYSOs) that are actively accreting and driving outflows: extended emission in the IRAC $4.5 \mu \mathrm{m}$ band, believed to trace shocked molecular gas. Maser studies of these extended $4.5 \mu \mathrm{m}$ sources (called EGOs, Extended Green Objects, for the common coding of 3-color IRAC images) have been and remain crucial for understanding the nature of EGOs. High detection rates in VLA $\mathrm{CH}_{3} \mathrm{OH}$ maser surveys provided the first proof that EGOs were indeed MYSOs driving outflows; our recent Nobeyama 45-m survey of northern EGOs shows that the majority are associated with $\mathrm{H}_{2} \mathrm{O}$ masers. Maser studies of EGOs also provide important constraints for the longstanding goal of a maser evolutionary sequence for MYSOs, particularly in combination with high resolution (sub)mm data. New SMA results show that Class I methanol masers can be excited by both young (hot core) and evolved (ultracompact HII region) sources within the same massive star-forming region.
\end{abstract}

Keywords. infrared: ISM- infrared: stars- ISM: jets and outflows- ISM: molecules- masersradio continuum: ISM- stars: formation- techniques: interferometric

\section{Introduction}

What are GLIMPSE Extended Green Objects (EGOs)? GLIMPSE-the Galactic Legacy Infrared Mid-Plane Survey Extraordinaire (Churchwell et al. 2009)-is a Spitzer Space Telescope survey of the Galactic Plane in the four bands of the Infrared Array Camera (IRAC; Fazio et al. 2004): 3.6, 4.5, 5.8, and $8.0 \mu \mathrm{m}$. The broad IRAC bands include emission features from a range of interstellar species (e.g. Fig. 1 of Reach et al. 2006). All of the IRAC bands include $\mathrm{H}_{2}$ lines; the $4.5 \mu \mathrm{m}$ band also includes the $\mathrm{CO}(v=1-0)$ bandhead and $\operatorname{Br} \alpha$. Notably, $4.5 \mu \mathrm{m}$ is also the only IRAC band to lack polycyclic aromatic hydrocarbon (PAH) emission features. This combination of characteristics means that in massive star-forming regions, shock-excited gas in (proto)stellar molecular outflows can stand out as morphologically distinct, extended $4.5 \mu \mathrm{m}$ emission. Thus, the ability to search for extended $4.5 \mu \mathrm{m}$ emission in GLIMPSE images presented an exciting opportunity to compile a new sample of candidate massive young stellar objects (MYSOs) 
with active outflows, independent of previous CO imaging surveys based largely on IRAS point sources (e.g. Zhang et al. 2001). Because the $4.5 \mu \mathrm{m}$ band is commonly coded as green in three-color IRAC images (RGB: 8.0, 4.5, 3.6 $\mu \mathrm{m}$ ), these extended $4.5 \mu \mathrm{m}$ sources are known as Extended Green Objects (EGOs; Cyganowski et al. 2008).

Cyganowski et al. (2008) cataloged over 300 EGOs in the GLIMPSE-I survey area $\left(10^{\circ} \leqslant|l| \leqslant 65^{\circ},|b| \leqslant 1^{\circ}\right)$. Based on their mid-infrared (MIR) colors and association with infrared dark clouds (IRDCs), Cyganowski et al. (2008) suggested that EGOs were specifically massive YSOs driving active outflows.

\section{The Nature of EGOs (as revealed by $\mathrm{CH}_{3} \mathrm{OH}$ masers)}

The first step, after the identification of EGOs as a class of objects by Cyganowski et al. (2008), was to test whether GLIMPSE EGOs were in fact massive YSOs driving outflows. To do this, Cyganowski et al. (2009) conducted sensitive, high-angular resolution searches for two diagnostic types of $\mathrm{CH}_{3} \mathrm{OH}$ masers towards a sample of EGOs with the Very Large Array (VLA) $\dagger: 6.7 \mathrm{GHz}$ Class II $\mathrm{CH}_{3} \mathrm{OH}$ masers, associated exclusively with massive YSOs (e.g. Minier et al. 2003) and $44 \mathrm{GHz}$ Class $\mathrm{I} \mathrm{CH}_{3} \mathrm{OH}$ masers, associated with molecular outflows and outflow-cloud interfaces (e.g. Kurtz et al. 2004). An initial sample of 28 EGOs was selected to (1) cover a range of MIR properties, including morphology, angular extent of $4.5 \mu \mathrm{m}$ emission, and the presence of 8 and/or $24 \mu \mathrm{m}$ counterparts, and (2) be visible from the northern hemisphere. Due to technical problems, however, there is a bias in the final survey sample: only 19 sources with (strong) $6.7 \mathrm{GHz}$ masers near the phase center were reobserved at $6.7 \mathrm{GHz}$, and only these 19 sources were observed at $44 \mathrm{GHz}$ (see Cyganowski et al. 2009 for details). As a result, the Cyganowski et al. (2009) sample is, in essence, a $6.7 \mathrm{GHz} \mathrm{CH}_{3} \mathrm{OH}$ maser-selected EGO subsample.

The detection rates for both $6.7 \mathrm{GHz}$ Class II and $44 \mathrm{GHz}$ Class I $\mathrm{CH}_{3} \mathrm{OH}$ masers were extremely high: $>64 \%$ (of the original 28 sources) and $~ 90 \%$ (of the 19 observed sources), respectively. For $6.7 \mathrm{GHz} \mathrm{CH}_{3} \mathrm{OH}$ masers, this detection rate was nearly twice that towards other MYSO samples (see also Cyganowski et al. 2009). The spatial distribution and kinematics of the two types of $\mathrm{CH}_{3} \mathrm{OH}$ masers are strikingly different. The $6.7 \mathrm{GHz}$ masers are centrally concentrated and usually coincident with $24 \mu \mathrm{m}$ emission, while the $44 \mathrm{GHz}$ masers are spatially distributed, often over tens of arcseconds, and coincident with $4.5 \mu \mathrm{m}$ emission. To complement the maser surveys and provide information about the thermal gas emission, Cyganowski et al. (2009) used the James Clerk Maxwell Telescope $(\mathrm{JCMT}) \ddagger$ to observe $\mathrm{HCO}^{+}(3-2), \mathrm{H}^{13} \mathrm{CO}^{+}(3-2)$, thermal $\mathrm{CH}_{3} \mathrm{OH}\left(5_{2,3}-4_{1,3}\right)$, and $\mathrm{SiO}(5-$ 4) emission. The velocities of the $44 \mathrm{GHz}$ masers cluster near the systemic velocity (as measured from the dense gas tracers observed with the JCMT), consistent with their excitation at interfaces between outflows and the surrounding molecular gas. In contrast, the velocities of the $6.7 \mathrm{GHz}$ masers exhibit every possible permutation with respect to the thermal gas $\mathrm{v}_{L S R}$ : different sources provide examples of $6.7 \mathrm{GHz}$ masers at and near the systemic velocity, only redshifted, only blueshifted, and both red and blueshifted but not at the systemic velocity. This diversity suggests that the $6.7 \mathrm{GHz}$ masers observed towards this EGO sample do not all arise in any single physical/dynamical structure.

The JCMT survey also provided further evidence, in addition to the Class I masers, that the target EGOs were associated with outflows. For all sources, the $\mathrm{HCO}^{+}$spectra

$\dagger$ The National Radio Astronomy Observatory operates the VLA and is a facility of the National Science Foundation operated under agreement by the Associated Universities, Inc.

$\ddagger$ The JCMT is operated by The Joint Astronomy Centre on behalf of the Science and Technology Facilities Council of the United Kingdom, the Netherlands Organisation for Scientific Research, and the National Research Council of Canada. 
showed broad line wings, and the detection rate for thermal $\mathrm{SiO}$ emission was very high: $90 \%$ (9 of a subset of 10 EGOs). Gas-phase $\mathrm{SiO}$ abundance is enhanced for only $\sim 10^{4}$ years after a shock (e.g. Pineau de Forets et al. 1997), so the SiO emission indicates that the outflows are being actively driven, as has been observationally confirmed in a survey comparing low-mass Class O and I sources (Gibb et al. 2004). High-resolution mm- $\lambda$ followup observations have confirmed the presence of bipolar molecular outflows in EGOs. Submillimeter Array (SMA) $\uparrow$ imaging of the EGOs G11.92-0.61 and G19.01-0.03 reveals high-velocity, well-collimated outflows traced by ${ }^{12} \mathrm{CO}(2-1)$ emission (Cyganowski et al. 2011a). The $\mathrm{CO}$ outflows are coincident with the extended $4.5 \mu \mathrm{m}$ emission in these sources, and many of the $44 \mathrm{GHz}$ Class $\mathrm{I} \mathrm{CH}_{3} \mathrm{OH}$ masers coincide with or trace the edges of the high-velocity $\mathrm{CO}$ outflow lobes.

In sum, the initial $\mathrm{CH}_{3} \mathrm{OH}$ maser studies of EGOs provided strong evidence that the survey targets were young MYSOs with active outflows, and so presumably ongoing accretion. High resolution observations of direct tracers of molecular outflows and hot cores have confirmed this picture for EGOs observed to date (Cyganowski et al. 2011a), and further high-resolution mm- $\lambda$ followup is ongoing.

It is worth emphasizing that extended $4.5 \mu \mathrm{m}$ emission acts as an effective way to find MYSOs in GLIMPSE, and that this is to an important extent a function of the shallowness of the GLIMPSE-I survey. Outflows in nearby low-mass star-forming regions exhibit extended $4.5 \mu \mathrm{m}$ emission in Spitzer images (e.g. Noriega-Crespo et al. 2004, Velusamy et al. 2007). However, the extended $4.5 \mu \mathrm{m}$ emission from such low-mass outflows is too faint to be detected in GLIMPSE (see also discussion in Cyganowski et al. 2008). In deeper Spitzer Galactic Plane surveys (such as the GLIMPSE-360 survey of the outer Galaxy, and the ongoing Deep GLIMPSE), extended $4.5 \mu \mathrm{m}$ emission will pick out outflows, including those from low- and intermediate- mass YSOs.

Millimeter Methanol Masers. The SMA observations of G11.92-0.61 and G19.01-0.03 also revealed probable $\mathrm{CH}_{3} \mathrm{OH}$ maser emission in the $229.759 \mathrm{GHz}$ Class I transition (Cyganowski et al. 2011a). The observed 229.759 GHz emission is spectrally narrow, and spatially and spectrally coincident with $44 \mathrm{GHz}$ masers. While the angular resolution of the SMA data is not sufficient to definitively establish masing based on brightness temperature, the 229.759/230.027 line ratios indicate nonthermal emission. Our ongoing SMA observations of EGOs indicate that $229.759 \mathrm{GHz}$ Class I masers may be common towards MYSO outflows. If so, such masers offer potential for self-calibrating very highresolution $1.3 \mathrm{~mm}$ observations of MYSOs, e.g. with ALMA.

Evolutionary State. Constraining the evolutionary state of EGOs is crucial for placing maser studies of EGOs in the broader context of maser studies of massive star-forming regions. In particular, the presence of an ultracompact (UC) HII region signals a comparatively late stage of massive star formation, when a young $\mathrm{O} / \mathrm{B}$ star has already ionized its environment. While it is important to remember that high resolution (sub)millimeter studies of UC HIIs typically reveal additional objects in a range of evolutionary stages (Hunter et al. 2008; Brogan et al. 2008; Hunter et al. 2004), the absence/presence of UC HIIs has been used in the past as a key divide between younger/older MYSO samples. To search for UC HIIs, shallow $44 \mathrm{GHz}(7 \mathrm{~mm})$ continuum data were obtained simultaneously during the $44 \mathrm{GHz}$ maser survey. The nondetection rate was $95 \%$, but the sensitivity was sufficient to rule out only bright UC HII regions (Cyganowski et al. 2009). To better constrain the cm- $\lambda$ emission of EGOs, Cyganowski et al. (2011b) carried out deep VLA

ब The Submillimeter Array is a joint project between the Smithsonian Astrophysical Observatory and the Academia Sinica Institute of Astronomy and Astrophysics and is funded by the Smithsonian Institution and the Academia Sinica. 
3.6 and $1.3 \mathrm{~cm}$ continuum observations of a sample of 14 EGOs associated with $6.7 \mathrm{GHz}$ $\mathrm{CH}_{3} \mathrm{OH}$ masers, $44 \mathrm{GHz} \mathrm{CH}_{3} \mathrm{OH}$ masers, or both. The surveys had angular resolution of $\sim 1^{\prime \prime}$ and $\sigma \sim 30 \mu \mathrm{Jy}_{\text {beam }}^{-1}(3.6 \mathrm{~cm})$ and $250 \mu \mathrm{Jy}^{\text {beam }}{ }^{-1}(1.3 \mathrm{~cm})$. The nondetection rate of these VLA surveys was $57 \%(8 / 14)$. The $\mathrm{cm}-\lambda$ EGO counterparts that were detected were generally weak $(\lesssim 1 \mathrm{mJy}$ at $3.6 \mathrm{~cm})$, and not detected at $1.3 \mathrm{~cm}$ due to the higher noise. Only 2 EGOs are associated with ultracompact or compact HII regions; both show $\mathrm{cm}-\lambda$ multiplicity, with morphological evidence that a less evolved source may be driving the $4.5 \mu \mathrm{m}$ outflow. One EGO is detected only at $1.3 \mathrm{~cm}$; comparison with $1.4 \mathrm{~mm}$ data suggests that the $1.3 \mathrm{~cm}$ emission is free-free from an optically thick hypercompact (HC) HII region (Cyganowski et al. 2011b). This EGO (G11.92-0.61) in fact hosts a protocluster of 3 compact mm continuum cores (Cyganowski et al. 2011a). The $1.3 \mathrm{~cm}$ source is associated with a $1.4 \mathrm{~mm}$ continuum core, hot core line emission, $24 \mu \mathrm{m}$ emission, and $\mathrm{H}_{2} \mathrm{O}$ and $6.7 \mathrm{GHz} \mathrm{CH}_{3} \mathrm{OH}$ masers.

\section{Masers in EGOs: Insights for masers in massive star forming regions}

In addition to constraining the nature of EGOs themselves, the wealth of multiwavelength data now available for many EGOs allows us to examine cross-correlations between different maser types and other star formation indicators (see also Brogan et al. 2011 and in this volume).

Class I $\mathrm{CH}_{3} \mathrm{OH}$ Masers as Evolutionary Indicators? The EGO G18.67+0.03 is particularly interesting in the context of proposed maser evolutionary sequences, which posit that the youngest MYSOs exhibit only Class $\mathrm{I} \mathrm{CH}_{3} \mathrm{OH}$ maser emission (e.g. Ellingsen 2006, Ellingsen et al. 2007, Breen et al. 2010). Our VLA $\mathrm{CH}_{3} \mathrm{OH}$ maser surveys revealed maser emission associated with 3 MIR sources in this region: two (including the EGO) have both $6.7 \mathrm{GHz}$ Class II and $44 \mathrm{GHz}$ Class I $\mathrm{CH}_{3} \mathrm{OH}$ masers, while the third MIR source has only $44 \mathrm{GHz}$ Class I masers (Cyganowski et al. 2009). The Class I maser-only MIR source has a $\mathrm{cm}-\lambda$ continuum counterpart, with properties consistent with a UC HII region (Cyganowski et al. 2011b). None of the other sources in the field have cm- $\lambda$ continuum emission in our deep VLA surveys. The association of $44 \mathrm{GHz} \mathrm{CH}_{3} \mathrm{OH}$ masers with a UC HII region in G18.67+0.03 is consistent with recent suggestions by Voronkov et al. (2010) that Class I masers may be excited by shocks driven by expanding HII regions. However, younger sources (such as hot cores) are often found in close proximity to UC HII regions (e.g. Hunter et al. 2006; Cyganowski et al. 2007 and references therein); therefore, additional multiwavelength information was needed. We conducted SMA $1.3 \mathrm{~mm}$ observations to determine the evolutionary states of the G18.67+0.03 maser sources based on their molecular line emission.

Our SMA observations reveal hot core line emission towards both Class II+Class I maser sources (Cyganowski et al. in prep.). In contrast, emission from only a few common species is detected towards the UC HII region/Class I maser-only source. All three maser sources are associated with molecular outflows traced by ${ }^{13} \mathrm{CO}(2-1)$ emission. However, the UC HII region outflow is not detected in $\mathrm{SiO}(5-4)$. As described above, $\mathrm{SiO}$ emission provides a discriminant between active ( $\mathrm{SiO}$ present) and "relic" or "fossil" outflows ( $\mathrm{SiO}$ absent). Thus, the lack of $\mathrm{SiO}$ emission is strong additional evidence that no younger YSO is present near the Class I-only maser source, and that these Class I masers are associated with the comparatively evolved UC HII region.

$\mathrm{H}_{2} \mathrm{O}$ maser (and $\mathrm{NH}_{3}$ ) Survey of Northern EGOs. Most maser studies of EGOs to date have focused on $\mathrm{CH}_{3} \mathrm{OH}$ masers. For the broader picture of masers in high-mass star forming regions, $\mathrm{H}_{2} \mathrm{O}$ masers in EGOs are an important missing piece of the puzzle. We 
have recently completed a Nobeyama $45-\mathrm{m}$ survey of all 94 northern EGOs $\left(\delta \gtrsim-20^{\circ}\right)$ for $\mathrm{H}_{2} \mathrm{O}$ maser and $\mathrm{NH}_{3}(1,1),(2,2)$, and $(3,3)$ emission (Cyganowski et al., in prep.). The median rms of the $\mathrm{H}_{2} \mathrm{O}$ maser survey is $\sim 0.11 \mathrm{Jy}$. The goals of this survey were (1) to evaluate the significance of the MIR categories from the Cyganowski et al. (2008) EGO catalog, (2) to compare the $\mathrm{H}_{2} \mathrm{O}$ maser properties of EGO subsamples (for example, those associated with Class I/II $\mathrm{CH}_{3} \mathrm{OH}$ masers), and (3) to compare $\mathrm{H}_{2} \mathrm{O}$ maser properties with clump physical properties from other tracers $\left(\mathrm{T}_{k i n}\right.$, density, etc.).

The overall $\mathrm{H}_{2} \mathrm{O}$ maser detection rate in our Nobeyama survey is $\sim 68 \%$. Cyganowski et al. (2008) classified EGOs as "likely" or "possible" outflow candidates based on MIR morphology; they also tabulated whether each EGO was or was not associated with an IRDC in the GLIMPSE images. The $\mathrm{H}_{2} \mathrm{O}$ maser detection rate is somewhat higher towards EGOs that are "likely" outflow candidates, and roughly comparable towards EGOs that are and are not associated with IRDCs. We find little evidence of statistically significant differences in the $\mathrm{H}_{2} \mathrm{O}$ maser properties (for example, the isotropic maser luminosity) of various EGO subsamples.

Recent studies have found correlations between the isotropic $\mathrm{H}_{2} \mathrm{O}$ maser luminosity and the properties of the driving source or surrounding clump. Urquhart et al. (2011) found a positive correlation between $\mathrm{H}_{2} \mathrm{O}$ maser luminosity and bolometric luminosity for both HII regions and MYSOs from the Red MSX Source (RMS) sample, while Breen \& Ellingsen (2011) report an anticorrelation between $\mathrm{H}_{2} \mathrm{O}$ maser luminosity and the $\mathrm{H}_{2}$ number density of the associated clump. Breen \& Ellingsen (2011) attribute this anticorrelation to an evolutionary effect, but caution that the clump densities were calculated assuming a single temperature for all clumps. Our Nobeyama survey and the $1.1 \mathrm{~mm}$ Bolocam Galactic Plane Survey (BGPS; Aguirre et al. 2011, Rosolowsky et al. 2010) together provide the data necessary to test this evolutionary interpretation and explore connections between $\mathrm{H}_{2} \mathrm{O}$ maser and clump properties: $\mathrm{H}_{2} \mathrm{O}$ maser spectra, $\mathrm{T}_{k i n}$ measurements from $\mathrm{NH}_{3}$, and clump properties from $1.1 \mathrm{~mm}$ dust continuum emission. Combining these datasets, we find no correlation between $\mathrm{H}_{2} \mathrm{O}$ maser luminosity and clump density. There is a weak correlation between $\mathrm{H}_{2} \mathrm{O}$ maser luminosity and clump temperature $\left(\mathrm{T}_{k i n}\right)$. This is consistent with the correlation of maser luminosity with bolometric luminosity found by Urquhart et al. (2011): the more luminous the central source, the more it will heat the surrounding clump.

\section{Conclusions}

In sum, maser studies have been instrumental in showing that GLIMPSE EGOs are associated with MYSOs driving active, massive outflows. Sensitive VLA surveys yielded exceptionally high detection rates for $\mathrm{CH}_{3} \mathrm{OH}$ Class I and II masers. In a somewhat less sensitive $(\sigma \sim 0.11 \mathrm{Jy})$ Nobeyama $45-\mathrm{m} \mathrm{H}_{2} \mathrm{O}$ maser survey, the detection rate is slightly higher than in similar searches towards other MYSO samples. By and large, $\mathrm{cm}$ continuum emission towards EGOs is weak, with most harboring an earlier phase of massive star formation than UC HII regions. Our SMA case study of G18.67+0.03 shows that Class $\mathrm{I} \mathrm{CH}_{3} \mathrm{OH}$ masers can be excited by both young (hot core) and older (UC HII) sources in the same massive star-forming region, indicating that simple evolutionary cartoons are probably not realistic. In our EGO $\mathrm{H}_{2} \mathrm{O}$ maser survey, we see no trends in $\mathrm{H}_{2} \mathrm{O}$ maser luminosity with clump density, association with IRDCs, or with $\mathrm{CH}_{3} \mathrm{OH}$ maser type (Class I or II or both). There is a weak trend in $\mathrm{H}_{2} \mathrm{O}$ maser luminosity with clump temperature, consistent with the $\mathrm{L}_{\mathrm{H}_{2} \mathrm{O}}$ vs. $\mathrm{L}_{\text {bol }}$ correlation. 


\section{Acknowledgements}

Support for this work was provided by NSF grant AST-0808119. C.J.C. was partially supported during this work by a National Science Foundation Graduate Research Fellowship, and is currently supported by an NSF Astronomy and Astrophysics Postdoctoral Fellowship under award AST-1003134.

\section{References}

Aguirre, J. E., Ginsburg, A. G., Dunham, M. K., et al. 2011, ApJS, 192, 4

Breen, S. L., Ellingsen, S. P., Caswell, J. L., \& Lewis, B. E. 2010, MNRAS, 401, 2219

Breen, S. L. \& Ellingsen, S. P. 2011, MNRAS, 416, 178

Brogan, C. L., Hunter, T. R., Cyganowski, C. J., et al. 2011, ApJ Letters, 739, L16

Brogan, C. L., Hunter, T. R., Indebetouw, R., et al. 2008, ApESSS, 313, 53

Churchwell, E., Babler, B. L., Meade, M. R., et al. 2009, PASP, 121, 213

Cyganowski, C. J., Brogan, C. L., \& Hunter, T. R. 2007, AJ, 134, 346

Cyganowski, C. J., Whitney, B. A., Holden, E., et al. 2008, AJ, 136, 2391

Cyganowski, C. J., Brogan, C. L., Hunter, T. R., \& Churchwell, E. 2009, ApJ, 702, 1615

Cyganowski, C. J., Brogan, C. L., Hunter, T. R., Churchwell, E., \& Zhang, Q. 2011a, ApJ, 729, 124

Cyganowski, C. J., Brogan, C. L., Hunter, T. R., \& Churchwell, E. 2011b, ApJ, 743, 56

Ellingsen, S. P. 2006, ApJ, 638, 241

Ellingsen, S. P., Voronkov, M. A., Cragg, D. M., et al. 2007, in: J. M. Chapman \& W. A. Baan (eds.), Astrophysical Masers 86 their Environments, Proc. IAU Symposium No. 242 (Cambridge, UK: Cambridge University Press), p. 213

Fazio, G. G., Hora, J. L., Allen, L. E., et al. 2004, ApJS, 154, 10

Gibb, A. G., Richer, J. S., Chandler, C. J., \& Davis, C. J. 2004, ApJ, 603, 198

Hunter, T. R., Brogan, C. L., Indebetouw, R., \& Cyganowski, C. J. 2008, ApJ, 680, 1271

Hunter, T. R., Brogan, C. L., Megeath, S. T., et al. 2006, ApJ, 649, 888

Hunter, T. R., Zhang, Q., \& Sridharan, T. K. 2004, ApJ, 606, 929

Kurtz, S., Hofner, P., \& Álvarez, C. V. 2004, ApJS, 155, 149

Minier, V., Ellingsen, S. P., Norris, R. P., \& Booth, R. S. 2003, A\&A A, 403, 1095

Noriega-Crespo, A., Morris, P., Marleau, F. R., et al. 2004, ApJS, 154, 352

Pineau des Forets, G., Flower, D. R., \& Chieze, J.-P. 1997, in: B. Reipurth \& C. Bertout (eds.), Herbig-Haro Flows and the Birth of Low-Mass Stars Proc. IAU Symposium No. 182 (Boston: Kluwer Academic Publishers), p. 199

Reach, W. T., Rho, J., Tappe, A., et al. 2006, AJ, 131, 1479

Rosolowsky, E., Dunham, M. K., Ginsburg, A., et al. 2010, ApJS, 188, 123

Urquhart, J. S., Morgan, L. K., Figura, C. C., et al. 2011, MNRAS, 418, 1689

Velusamy, T., Langer, W. D., \& Marsh, K. A. 2007, ApJ Letters, 668, L159

Voronkov, M. A., Caswell, J. L., Ellingsen, S. P., \& Sobolev, A. M. 2010, MNRAS, 405, 2471

Zhang, Q., Hunter, T. R., Brand, J., et al. 2001, ApJ Letters, 552, L167 